\title{
分析胺磑酮、厄贝沙坦联合治疗慢性心力衰竭伴室心律 失常的临床效果
}

\author{
张跃军* \\ 张家口市下花园区医院，河北 075300
}

\begin{abstract}
摘 要: 目的：对HF伴室心律失常患者联合应用胺碘酮+厄贝沙坦治疗, 并分析其临床效果。方法：选取2018 年11月一2020年10月, 在我院治疗的88例 HF伴室心律失常患者, 将其分为两组。对照组44例, 使用胺碘酮治疗; 观 察组44例, 在此基础上, 联用厄贝沙坦治疗。结果: 治疗后, 观察组患者的LVEF、每分输出量明显高于对照组, LVESD明显小于对照组 $(P<0.05)$; 观察组患者治疗1个月、3个月、6个月的窦性心律维持率均明显高于对照组 $(P$ $<0.05)$; 观察组患者不良反应率为 $13.64 \%$, 对照组为 $18.18 \%$, 无明显差异 $(P>0.05)$ 。结论: 对HF伴室心律失常 应用胺碘酮十厄贝沙坦治疗可更好地改善患者心功能，维持其棠性心律，且安全性良好。
\end{abstract}

关键词：慢性心力衰竭；室心律失常；厄贝沙坦；胺碘酮

\section{The Clinical Effect of Amiodarone and Irbesartan in the Treatment of Chronic Heart Failure with Ventricular Arrhythmia}

\author{
Yue-Jun Zhang* \\ Zhangjiakou Xiahuayuan District Hospital, Zhangjiakou 075300, Hebei, China
}

\begin{abstract}
Objective: To analyze the clinical effect of amiodarone combined with irbesartan in HF patients with ventricular arrhythmia. Method: From November 2018 to October 2020, 88 HF patients with ventricular arrhythmia in our hospital were selected and divided into two groups. 44 cases in the control group were treated with amiodarone; 44 cases in the observation group were treated with irbesartan on this basis. Result: After treatment, the LVEF and output per minute of the observation group were significantly higher than those of the control group, and the LVESD was significantly lower than that of the control group $(P<0.05)$; the sinus rhythm maintenance rate of the observation group was significantly higher than that of the control group $(P<0.05)$; the adverse reaction rate of the observation group was $13.64 \%$, and that of the control group was $18.18 \%$, with no significant difference $(P>0.05)$. Conclusion: Amiodarone combined with irbesartan in the treatment of HF with ventricular arrhythmia can better improve the cardiac function of patients, maintain its sinus rhythm, and has good safety.
\end{abstract}

Keywords: Chronic heart failure; ventricular arrhythmia; Irbesartan; Amiodarone

\section{一、前言}

慢性心力衰竭 ( HF ) 是心内科常见疾病, 主要发病群体为中老年人。该病主要为心脏疾病、心血管病变长期进展 所致, 由于心室负荷增加, 心脏收缩、舒张能力下降, 心脏㬌血能力受到影响, 回心血量不足, 难以维持正常心脏循 环, 最终形成 $\mathrm{HF}^{[1]}$ 。随着 $\mathrm{HF}$ 病情的进展, 患者可出现心律失常, 威胁其生命安全。在该类患者临床治疗时, 不仅需要 缓解HF症状、延缓病情进展, 还需要纠正心律失常 ${ }^{[2]}$ 。目前, 临床上常用的抗心律失常药物较多, 但大部分药物均具 有一定的降低心肌收缩的作用, 可能导致患者心脏洜血能力进一步下降, HF病情加重。因此, 在选择临床用药时应更 加谨慎。胺碘酮是一种常用的肾上腺素术受体拮抗剂类抗心律失常药物, 与其他抗心律失常药物相比, 其负性肌力作 用更低。而厄贝沙坦属于血管紧张素 II ( Ang II ) 受体拮抗剂 ( ARB ) , 在扩张血管、降低心肌耗氧、恢复心室重构

*通讯作者：张跃军，1977年12月，男，汉族，河北张家口人，现任张家口市下花园区医院内科医师，副主任医 师, 本科。研究方向: 心脑血管疾病。 
方面具有较好的效果 ${ }^{[2]}$ 。因此, 本文将对HF伴室心律失常患者联合应用胺碘酮+厄贝沙坦治疗, 并分析其临床效果, 现报道如下。

\section{二、资料与方法}

(一) 一般资料

选取2018年11月-2020年10月，在我院治疗的88例HF伴室心律失常患者。

1. 纳人标准

(1) 参考 《内科学》确诊为 HF 、心律失常。

(2) 心功能分级 (NYHA) 为 III $\sim \mathrm{IV}$ 级。

(3) 左心室射血分数 ( LVEF) $\leqslant 40 \%$ 。

（4）已签署知情同意书。

2. 排除标准

（1）其他原因导致心律失常患者，例如中毒、先天性心脏结构异常等。

(2) 肝肾功能不全患者。

(3) 合并肺部疾病患者。

（4）急性期心脑血管疾病患者。

( 5 ）预计生存期低于 6 个月患者。

(6) 对本次研究使用药物过敏患者等。

采取随机数字表法, 将其分为两组。观察组44例, 男23例, 女21例, 年龄52 79岁, 平均 (68.59 4.49) 岁, NYHA分级：III 级19例, IV 级25例; 原发疾病: 冠心病15例, 高血压性心脏病17例, 心肌病4例, 室性早搏8例。对照 组44例, 男24例, 女20例, 年龄53 80岁, 平均 (68.97 \pm 5.01$)$ 岁, NYHA分级: III级18例, IV 级26例; 原发疾病: 冠 心病16例, 高血压性心脏病15例, 心肌病4例, 室性早搏9例。两组患者上述资料无显著差异 $(P>0.05)$ 。本次研究 已经过伦理委员会审核。

\section{(二) 方法}

两组患者的常规治疗措施相同, 包括控制血压、调脂、抗凝、抗血小板以及常规抗心衰治疗。在此基础上, 对照 组患者使用胺碘酮 (国药准字H50020463) 治疗, 口服, 初始剂量 $600 \mathrm{mg} / \mathrm{d}, 1$ 周后调整为 $400 \mathrm{mg} / \mathrm{d}$, 治疗 1 周后, 按照 $200 \mathrm{mg} / \mathrm{d}$ 的剂量维持治疗, 共治疗 6 个月。

观察组患者在此基础上, 使用厄贝沙坦 ( 国药准字 $\mathrm{H} 20203313$ ) 治疗, 口服, 初始剂量 $150 \mathrm{mg} / \mathrm{d}$, 随后根据其血 压水平调整，治疗6个月。

(三) 评价标准

1. 对比两组患者的心功能指标改善情况，包括LVEF、左心室收缩末期内径（LVESD）、每分输出量。

2. 对比两组患者用药后不同阶段（治疗后1个月、3个月、6个月）的窦性心律维持率。

3. 对比两组患者的不良反应情况。

( 四) 统计学方法

应用SPSS22.0处理数据, 计数资料、计量资料以 $(\%) 、(\bar{x} \pm s)$ 表示, 采用 $X^{2} 、 t$ 检验。 $P<0.05$ 表示差异, 有 统计学意义。

\section{三、结果}

表1 两组患者心功能指标对比 $(n=44)$

\begin{tabular}{ccccccc}
\hline \multirow{2}{*}{ 组别 } & \multicolumn{2}{c}{ LVEF $(\%)$} & \multicolumn{2}{c}{$\operatorname{LVESD}(\mathrm{mm})$} & \multicolumn{2}{c}{ 每分输出量 $(\mathrm{L} / \mathrm{min})$} \\
\cline { 2 - 6 } & 治疗前 & 治疗后 & 治疗前 & 治疗后 & 治疗前 & 治疗后 \\
\hline 观察组 & $37.28 \pm 1.67$ & $50.57 \pm 1.06$ & $57.79 \pm 3.03$ & $51.06 \pm 2.25$ & $3.13 \pm 0.24$ & $4.72 \pm 0.22$ \\
对照组 & $37.78 \pm 1.75$ & $45.16 \pm 1.11$ & $57.88 \pm 2.89$ & $55.56 \pm 2.36$ & $3.14 \pm 0.27$ & $4.18 \pm 0.24$ \\
$t$ & 1.371 & 23.381 & 0.143 & 9.154 & 0.186 & 11.002 \\
$P$ & $>0.05$ & $<0.05$ & $>0.05$ & $<0.05$ & $>0.05$ & $<0.05$ \\
\hline
\end{tabular}


（一）心功能指标改善情况对比

治疗后，观察组患者的LVEF、每分输出量明显高于对照组，LVESD明显小于对照组 $(P<0.05)$ ，见表1。

(二) 窦性心律维持率对比

观察组患者治疗 1 个月、3个月、6个月的窦性心律维持率分别为 $72.73 \% 、 84.09 \% 、 79.55 \%$, 对照组分别为 $47.73 \% 、 63.64 \% 、 63.64 \% ，$ 差异明显（ $P<0.05 ） ， 见$ 表 2 。

表2 两组患者窦性心律维持率对比 $[n(\%)]$

\begin{tabular}{ccccc}
\hline 组别 & 例数 & 治疗1个月 & 治疗3个月 & 治疗6个月 \\
\hline 观察组 & 44 & $32(72.73)$ & $37(84.09)$ & $35(79.55)$ \\
对照组 & 44 & $21(47.73)$ & $28(63.64)$ & $26(59.09)$ \\
$X^{2}$ & - & 5.74 & 4.77 & 4.33 \\
$P$ & - & $<0.05$ & $<0.05$ & $<0.05$ \\
\hline
\end{tabular}

(三) 不良反应对比

观察组患者出现胃肠道反应 3 例, 窦性心动过缓 2 例, QT间期延长 1 例, 发生率 $13.64 \%$; 对照组患者出现胃肠道反 应3例，窦性心动过缓3例， QT间期延长2例，发生率18.18\%，无明显差异（ $\left.X^{2}=0.34 ， P>0.05\right) 。$

四、讨论

$\mathrm{HF}$ 是一种常见的危重症, 据相关调查显示, HF患者5年生存率与恶性肿瘤相近, 5年死亡率甚至超过恶性肿瘤, 重症 $\mathrm{HF}$ 患者 1 年死亡率约为 $50 \%$ 。近年来, 随着我国人口结构的改变, 老年人口增加, HF发生率也显著提高, 且患者 多伴有室心律失常, 两者相互促进, 增加了治疗难度 ${ }^{[3]}$ 。目前, 临床上对HF伴室心律失常的治疗目标主要为缓解心衰 症状、改善心功能、纠正心律失常 ${ }^{[4]}$ 。

从HF的病理机制上看, 心室重构是其主要病理基础, 而心室重构的诱因较多, 细胞外基质病变、肾素-血管紧张素醛固酮（RAAS）系统过度激活、Ang II 水平升高均会诱发心室重构。而心肌长期处于失代偿态, 则会诱发心律失常 ${ }^{[3]}$ 。 胺碘酮为苯呋喃类衍生物, 兼具IV类、II类、I类抗心律失常药物的特性, 可扩张冠状动脉血流, 选择性较高, 其作用机 制与 $\beta$ 受体阻滞剂相似, 可抑制钾离子外流, 改变心肌组织电位, 从而达到消减折返激动、缓解室性早搏及心动过度、 降低窦房结自律性、减慢其传导速度的治疗效果。同时, 该药负性肌力作用较小, 更适合HF患者的治疗 ${ }^{[6]}$ 。厄贝沙坦为 $\mathrm{ARB}$ 类药物, 具有较高的特异性, 给药可迅速吸收, 生物利用率可达到 $80 \%$, 可阻断醛固酮释放, 抑制血管收缩, 达到 逆转心室重构的目的 ${ }^{[7]}$ 。以往有研究显示 ${ }^{[8]}$, 对HF伴室心律失常患者联合应用上述两种药物治疗可显著改善患者心功能 指标以及RAAS指标，明显优于单用胺碘酮治疗。

在本次研究中, 治疗后, 观察组患者的LVEF、每分输出量明显高于对照组, LVESD明显小于对照组 $(P<$ $0.05)$; 观察组患者治疗1个月、3个月、6个月的窦性心律维持率均明显高于对照组 $(P<0.05)$, 可见两者联用的治 疗效果更好, 分析其原因, 主要是由于厄贝沙坦可阻断血管紧张素与受体结合, 有利于胺碘酮发挥疗效, 两者联用可 同时发挥抗心律失常、抑制心室重构的作用。同时, 观察组患者不良反应率为 $13.64 \%$, 对照组为 $18.18 \%$, 无明显差 异 $(P>0.05)$, 提示两者联用安全良好, 主要是由于厄贝沙坦作用平稳, 可经葡萄酸醛化代谢或氧化代谢, 因此不 会增加不良反应 ${ }^{[9-10]}$ 。

综上所述, 对HF伴室心律失常应用胺碘酮+厄贝沙坦治疗可更好地改善患者心功能, 维持其窦性心律, 且安全性 良好。

\section{参考文献:}

[1]刘超群, 张军. 厄贝沙坦联合胺碘酮治疗心力衰竭合并心律失常患者的疗效及对心功能的影响分析 [J]. 中国药物与 临床, 2020,20(17):2933-2935.

[2]孙鹭.胺碘酮与厄贝沙坦联合治疗心力衰竭合并心律失常的疗效及对患者心功能的影响 [J]. 中国实用医药, 2020,15(19):112-113.

[3]廖振城. 分析胺碘酠联合厄贝沙坦治疗心力衰竭合并心律失常的疗效及其对患者心功能的影响 [J]. 中国现代药物 应用, 2020,14(12):124-126。

[4]胡鹏里. 胺碘酮结合厄贝沙坦治疗慢性心力衰竭合并室性心律失常的效果评估 [J]. 心血管病防治知识(学术版), 
2020,10(02):29-31.

[5]张鹏.胺碘酮联合厄贝沙坦治疗心力衰竭合并心律失常的疗效探讨[J].当代医药论丛, 2020,18(01):120-121.

[6]康玲玲. 厄贝沙坦联合胺碘酮治疗心力衰竭合并房颤的疗效及其对心功能、血浆Ang- II N NE及BNP水平的影响 [J]. 心血管康复医学杂志, 2019,28(06):760-764.

[7]程丽丹.胺碘酮联合厄贝沙坦治疗心力衰竭合并心律失常临床疗效[J].现代诊断与治疗, 2019,30(24):4332-4333.

[8]齐婷婷. 胺磑酮联合厄贝沙坦治疗心力衰竭并心律失常的临床疗效及对患者心功能的影响 [J]. 世界最新医学信息 文摘, 2019,19(86): 158+163.

[9]刘旭.厄贝沙坦联合胺碘酮治疗慢性心力衰竭合并室性心律失常的临床疗效分析[J].基层医学论坛, 2019,23(28): 4067-4068.

[10]刘文斌,罗丽娟,戴显娇.胺碘酮联合厄贝沙坦对心力衰竭合并心律失常的临床疗效及心率变异性和炎症因子的 影响[J]. 医学理论与实践, 2019,32(16):2545-2546. 